\title{
Identification of D-amino acid oxidase and propiverine interaction partners and their potential role in the propiverine-mediated nephropathy
}

\author{
Marcia Y. Maier ${ }^{\mathrm{a}, \mathrm{b}, 1}$, Lisanne Luks ${ }^{\mathrm{a}, 1}$, Oliver R. Baudendistel ${ }^{\mathrm{b}, \mathrm{c}}$, Valentin Wittmann ${ }^{\mathrm{b}, \mathrm{c}}$, \\ Daniel R. Dietrich ${ }^{\mathrm{a}, \mathrm{b}, *}$

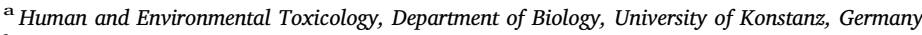 \\ ${ }^{\mathrm{b}}$ Konstanz Research School Chemical Biology (KoRS-CB), University of Konstanz, Germany \\ ${ }^{\mathrm{c}}$ Organic Chemistry/Bioorganic Chemistry, Department of Chemistry, University of Konstanz, Germany
}

\begin{abstract}
A B S T R A C T
Propiverine, a frequently-prescribed pharmaceutical for the treatment of symptoms associated with overactive bladder syndrome, provoked massive intranuclear and cytosolic protein inclusions in rat proximal tubule epithelium, primarily consisting of the peroxisomal targeting signal 1 (PTS1) containing protein D-amino acid oxidase (DAAO). As this type of nephropathy was also observed for other drugs, the aim was to determine whether propiverine interferes with trafficking and/or import of peroxisomal proteins. To elucidate this, DAAOand propiverine-specific interaction partners from human HEK293 and rat WKPT cell lines and rat kidney and liver homogenate were determined using co-immunoprecipitation with subsequent nano-ESI-LC-MS/MS analyses. Corroboration of the role of DAAO- and/or propiverine-specific interaction partners in the drug-induced DAAO accumulation was sought via specific immunofluorescence staining of rat kidney sections from control and propiverine-treated rats. Above analyses demonstrated the interaction of propiverine with several protein classes, foremost peroxisomal proteins (DAAO, MFE2, HAOX2) and proteins of the protein quality control system, i.e. chaperones (HSP70 and DnaJ co-chaperones), proteases and proteasomal proteins (regulatory subunits of the $26 \mathrm{~S}$ proteasome; Rpn1/2). The immunofluorescence analysis revealed mislocalization of many PTS1-proteins (DAAO, CAT, MFE2, ACOX1, EHHADH) in rat renal sections, strongly suggesting that propiverine primarily binds to PTS1 proteins resulting in the formation of PTS1 but not PTS2 or peroxisomal membrane protein (PMP) accumulations. Moreover, chaperones involved in peroxisomal trafficking (HSC70, DnaJB1) and peroxisomal biogenesis factor proteins (PEX3, PEX5, PEX7), also presented with distinct mislocalization patterns. Concomitantly, an increased number of peroxisomes was observed, suggestive of a compensatory mechanism for the presumably suboptimally functioning peroxisomes.

Overall, the data presented suggested that propiverine interacts exclusively with DAAO or with a selected number of PTS1 proteins. The consequence of this interaction is the abrogated trafficking and peroxisomal import of PTS1 proteins concomitant with their nuclear and cytosolic accumulation due to inhibited degradation and imbalanced protein homeostasis.
\end{abstract}

\section{Introduction}

Unexpected intranuclear and cytosolic protein accumulations in rat proximal tubule epithelial cells appear to be a reoccurring phenomenon in non-clinical safety studies [1-5]. Propiverine, a frequently-prescribed pharmaceutical for the treatment of symptoms associated with overactive bladder syndrome [6], is one example for a drug causing this type of protein accumulation $[3,7,8]$. The most abundant protein within these accumulations was identified as D-amino acid oxidase (DAAO) [3], a peroxisomal enzyme necessary for the degradation of D-amino acids [9]. Although, a priori, this protein accumulation phenomenon was considered rat-specific and of no human relevance [3], the concomitant mislocalization of a peroxisomal protein within the nucleus and cytosol appeared exceptional and warrants further investigation. Indeed, although multiple targeting of some mammalian peroxisomal proteins exists, none were previously reported to be present in the

Abbreviations: hDAAO, rDAAO, human and rat D-amino acid oxidase; PTS1, peroxisomal targeting signal 1; PTS2, peroxisomal targeting signal 2; PMPs, peroxisomal membrane proteins; PEX, peroxin; HEK293, human embryonic kidney; WKPT, Wistar-Kyoto rat kidney proximal tubule

* Corresponding author. Universitätsstrasse 10, 78457 Konstanz, Germany.

E-mail address: daniel.dietrich@uni-konstanz.de (D.R. Dietrich).

$\mathbf{1}$ These authors contributed equally to this work. 
nucleus [10].

Since no direct interaction of propiverine or its major metabolite, propiverine- $N$-oxide, with DAAO was observed, yet a complete loss of DAAO's peroxisomal localization was reported in previous studies [11], the question was raised whether propiverine interferes with the correct protein folding, shuttling and/or import of DAAO into peroxisomes. Indeed, recent in vitro analyses of cyto-nuclear shuttling of human and rat DAAO demonstrated that interference with the peroxisomal transport, i.e. deletion of the PTS1 sequence or PEX5 knockdown, resulted in cytosolic DAAO localization as well as passive diffusion into the nucleus [12]. The latter supports the hypothesis that propiverine and/or its metabolite(s) may interfere at the level of proteins intricately involved in DAAO trafficking from its cytosolic synthesis to the correct integration into the peroxisome [12].

Like all peroxisomal membrane and matrix proteins, DAAO is synthesized ribosomally, folded in the cytosol under chaperone control and post-translationally imported into peroxisomes [13]. Proper folding is mediated by direct binding of several chaperones including T-complex protein 1 (TCP1), HSP/HSC70 and HSP40 to newly emerging peroxisomal proteins and necessary for peroxisomal import [14-17]. DAAO contains a C-terminal peroxisomal targeting signal type 1 (PTS1) and is therefore recognized by the cytosolic PEX5 receptor that guides PTS1 cargos to the peroxisomal membrane [18]. At the membrane, the fully folded PTS1 cargo translocates across the peroxisomal membrane by interacting with several transmembrane peroxin (PEX) proteins i.e. the peroxisomal docking/translocation complex [19]. In contrast to the PTS1-containing proteins, a small number of peroxisomal proteins contain a N-terminal PTS2 sequence which is recognized by the cytosolic PEX7 receptor, thus shuttled and imported - together with the long isoform of PEX5 (PEX5L) - into the peroxisomal matrix [20]. Peroxisomal membrane proteins (PMPs) belonging to type I PMPs, on the other hand, are shuttled and imported into peroxisomes via the cytosolic PEX19 receptor which docks to the membrane-bound PEX3 (or PEX16 in mammalian cells) [21]. In contrast, the subclass of type II PMPs, e.g. PEX3, reaches peroxisomes via a PEX19/16/3-independent import route starting from the ER via vesicle-mediated trafficking [21]. Type II PMPs are either post- or co-translationally inserted into the ER membrane using the same machinery as secretory proteins [22] followed by subsequent budding from the ER to form preperoxisomal vesicles [23] which are also the basis for de novo peroxisome biogenesis [21]. Thus, any form of abrogated peroxisomal biogenesis and/or integration of peroxisomal proteins can lead to varying degrees of nonfunctionality of peroxisomes summarized as peroxisome biogenesis disorders (PBDs) [24]. In view of the fact that propiverine-induced protein inclusions were found to be developing slowly and were reversible upon cessation of drug exposure, but were not associated with overt renal toxicity in vivo $[3,7,8]$, a subtle but specific interference of propiverine, e.g. with DAAO trafficking, is considered a more likely mechanistic explanation for the observed phenomenon rather than a general disturbance of cell homeostasis. Moreover, the question was raised whether propiverine treatment results in a more generalized dysregulation of peroxisomal protein trafficking and import, similar to a PBD, rather than being restricted to interference with DAAO homeostasis only. To elucidate the latter a two-pronged approach was taken: A) Identification of DAAO-specific interaction partners in human HEK293 and rat WKPT renal cells using a co-immunoprecipitation (CoIP) method coupled to nano-LC-ESI-MS/MS for protein identification, and $B$ ) Identification of propiverine-specific interaction partners from rat kidney cortex homogenate using a specifically synthetized biotincoupled propiverine derivative for Co-IP coupled to nano-LC-ESI-MS/ MS. Finally, corroboration of the role of DAAO- and/or propiverinespecific interaction partners in the drug-induced DAAO accumulation was sought via specific immunofluorescence staining of rat kidney sections from control and propiverine-treated rats.

\section{Material and methods}

\subsection{Cell culture}

Mammalian pEYFP-C3, pEYFP-rDAAO-C3 and pEYFP-hDAAO-C3 expression vectors [25-27] were used for overexpression of EYFP or EYFP-DAAO (DAAO ${ }_{\text {perox }}$ ) in HEK293 cells. With the exception of EYFP$\mathrm{r} / \mathrm{hDAAO}_{\text {cyt }}(\triangle \mathrm{PTS}$ mutants), only stably transfected, monoclonal cell lines were used for all ensuing experiments. To produce $\triangle$ PTS1 mutants of rDAAO and hDAAO (DAAO ${ }_{\text {cyt }}$ ), site-directed mutagenesis was performed as previously described [12] and cells were subsequently transiently transfected with FuGENE [12]. HEK293 (human embryonic kidney) cells were purchased from the Leibniz Institute DSMZ - German Collection of Microorganisms and Cell Cultures and cultured in Dulbecco's modified Eagle's medium (DMEM) containing low glucose ( $1 \mathrm{~g} /$ L) supplemented with $10 \%(\mathrm{v} / \mathrm{v})$ fetal bovine serum (FBS) gold, $100 \mathrm{U} /$ $\mathrm{mL}$ penicillin and $0.1 \mathrm{mg} / \mathrm{mL}$ streptomycin. WKPT (WKY rat, kidney proximal tubule) cells were kindly provided by Prof. Dr. Frank Thevenod (University of Witten/Herdecke, Germany) and were cultured in DMEM Nutmix F12 (Ham) containing 10\% (v/v) FBS, $100 \mathrm{U} / \mathrm{mL}$ penicillin and $0.1 \mathrm{mg} / \mathrm{mL}$ streptomycin, $1.2 \mathrm{mg} / \mathrm{mL} \mathrm{NaHCO}, 5 \mu \mathrm{g} / \mathrm{mL}$ insulin, $4 \mu \mathrm{g} / \mathrm{mL}$ dexamethasone, $0.01 \mu \mathrm{g} / \mathrm{mL} \mathrm{EGF}$, and $5 \mu \mathrm{g} / \mathrm{mL}$ apotransferrin. All cell lines were routinely tested for mycoplasma contamination (GATC).

\subsection{Co-immunoprecipitation of DAAO interaction partners from HEK293 cells}

Since none of the tested, established human renal cell lines (e.g. RPTEC/TERT1) expressed endogenous DAAO, HEK293 cells were transfected with DAAO and were proven to be highly suitable to study peroxisomal trafficking and protein homeostasis as shown earlier [12]. Indeed, recent data confirmed that peroxisomes of HEK293 cells reacted in a comparable manner to rat proximal tubule epithelial cell peroxisomes in vivo (Maier et al., 2017, unpublished data). Accordingly, coimmunoprecipitation of EYFP-DAAO interaction partners was performed using GFP-Trap_MA (Chromotek, \#gtm-20) according to manufacturer's recommendations. Briefly, cells were seeded at 4E4 cells/ $\mathrm{cm}^{2}$ and harvested after $72 \mathrm{~h}$. Three confluent $10 \mathrm{~cm}^{2}$ dishes were used for one experiment with stably transfected cells (EYFP, EYFP-r/ $\mathrm{hDAAO}_{\text {perox }}$ ) and five $10 \mathrm{~cm}^{2}$ dishes were used for transiently transfected cells (EYFP-r/hDAAO ${ }_{\text {cyt }}$ ) to obtain similar protein yields. All subsequent steps were performed on ice. For harvesting, cells were washed twice with cold PBS and lyzed in $1 \mathrm{~mL}$ lysis buffer (10 mM Tris$\mathrm{HCl} \mathrm{pH} \mathrm{7.5,} 150 \mathrm{mM} \mathrm{NaCl}, 0.5 \mathrm{mM}$ EDTA, 0.5\% NP-40, $1 \mathrm{mM}$ PMSF, $1 \times$ Halt $^{\mathrm{TM}}$ Protease Inhibitor Cocktail) by passing five times through a $27 \mathrm{G}$ needle. The lysate was centrifuged for $10 \mathrm{~min}$ at $20.000 \mathrm{~g}$ and $4{ }^{\circ} \mathrm{C}$ and the supernatant diluted in $300 \mu \mathrm{L}$ dilution buffer $(10 \mathrm{mM}$ Tris- $\mathrm{HCl}$ pH 7.5, $150 \mathrm{mM} \mathrm{NaCl}, 0.5 \mathrm{mM}$ EDTA, $1 \mathrm{mM}$ PMSF, $1 \times$ Halt $^{\mathrm{tm}}$ Protease Inhibitor Cocktail). Subsequently, the diluted lysate was added to $25 \mu \mathrm{L}$ preequilibrated beads and placed on an overhead shaker at $4^{\circ} \mathrm{C}$ over night. The following day, beads were washed six times using dilution buffer and proteins eluted in two consecutive steps by boiling for $10 \mathrm{~min}$ in $95^{\circ} \mathrm{C}$ in $2 \times$ SDS buffer. Eluates were combined, loaded on a $12 \%$ SDS-gel and whole lanes were subjected to nano-LC-ESI-MS/MS analysis.

\subsection{Pull-down of biotin-linked propiverine interaction partners}

To identify propiverine-specific interaction partners from rat kidney and liver homogenate, Pierce ${ }^{\mathrm{TM}}$ Streptavidin Magnetic Beads (Thermo Scientific, \#88816) were used according to manufacturer's recommendations. Male Wistar rats were fumigated and bled to death from the cervical artery. Rat kidney homogenate was prepared from both kidney cortexes and liver homogenate from tissue derived from different parts of all four lobes. All subsequent steps were performed on 
ice. Homogenates were prepared using lysis buffer $(10 \mathrm{mM}$ Tris-HCl, $150 \mathrm{mM}$ NaCl, $0.5 \mathrm{mM}$ EDTA, 0.1\% Tween20, $1 \mathrm{mM}$ PMSF, $1 \times$ Halt $^{\mathrm{TM}}$ Protease Inhibitor Cocktail) in a tissue grinder and subsequent passage through a $22 \mathrm{G}$ needle. Lysate was centrifuged at $4000 \mathrm{~g}$ for $15 \mathrm{~min}$ at $4{ }^{\circ} \mathrm{C}$ and subsequently protein concentration was determined using Pierce BCA Protein Assay Kit (Thermo Scientific). Lysates were diluted in $300 \mu \mathrm{L}$ wash/binding buffer ( $25 \mathrm{mM}$ Tris- $\mathrm{HCl}, \mathrm{pH} 7.2,150 \mathrm{mM} \mathrm{NaCl}$, $0.1 \%$ Tween $20,1 \mathrm{mM}$ PMSF, $1 \times$ Halt $^{\mathrm{TM}}$ Protease Inhibitor Cocktail) to a final concentration of $3 \mu \mathrm{g} / \mu \mathrm{L}$ total protein. Finally, $1750 \mathrm{pmol}$ of biotin-propiverine derivative 11 (Fig. 2 and Supplementary Information) or D-biotin 9 was added and incubated overnight at $4{ }^{\circ} \mathrm{C}$ on a rotating platform. The following day, samples were added to $50 \mu \mathrm{L}$ preequilibrated magnetic beads. After $2 \mathrm{~h}$ immobilization of biotin-propiverine derivative 11 or D-biotin $\mathbf{9}$, beads were collected on a magnetic stand and supernatant, i.e. flow-through, was removed. Beads were washed three times with $300 \mu \mathrm{L}$ wash/binding buffer to remove nonspecific interacting proteins. In a first elution step, beads were incubated for 5 min at RT using $50 \mu \mathrm{L}$ of a mild, high-salt elution buffer

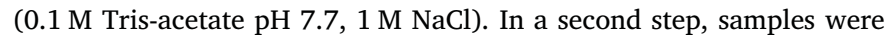
boiled for $5 \mathrm{~min}$ at $90^{\circ} \mathrm{C}$ in $50 \mu \mathrm{L}$ EDTA-formamide elution buffer (10 mM EDTA pH 8.2, 95\% formamide).

\subsection{Protein identification via nano-LC-ESI-MS/MS}

Eluates derived from EYFP-DAAO Co-IP or propiverine pull-down were separated on a $12 \%$ SDS-PAGE for $10 \mathrm{~min}$. Then, lanes were cut out and analyzed at the Proteomics Center, University of Konstanz using nano-LC-ESI-MS/MS. All samples were reduced with DTT ( $30 \mathrm{~min}, 56^{\circ} \mathrm{C}$ ) and alkylated with chloroacetamide (60 min, RT). Digestions were performed using trypsin $\left(4 \mathrm{~h}, 37^{\circ} \mathrm{C}\right)$. The digests were analyzed on an Orbitrap Fusion Tribrid mass spectrometer (Thermo Fisher Scientific, Bremen, Germany) interfaced with an Easy-nLC 1200 nanoflow liquid chromatography system (Thermo Scientific, Odense, Denmark). The peptide digests were reconstituted in $0.1 \%$ formic acid and loaded onto the analytical column $(75 \mu \mathrm{m} \times 15 \mathrm{~cm})$. Peptides were resolved at a flow rate of $300 \mathrm{~nL} / \mathrm{min}$ using a linear gradient of $5-35 \%$ solvent B $(0.1 \%$ formic acid in acetonitrile) over $45 \mathrm{~min}$. Data-dependent acquisition with full scans in 350-1500 $\mathrm{m} / \mathrm{z}$ range was carried out using the Orbitrap mass analyzer at a mass resolution of 120.000 at $200 \mathrm{~m} / \mathrm{z}$. Most intense precursor ions were selected at top speed data dependent mode with a maximum cycle time of $3 \mathrm{~s}$. Peptides with charge states 2-5 were selected, and dynamic exclusion was set to $30 \mathrm{~s}$. Precursor ions were fragmented using higher-energy collision dissociation (HCD) set to $35 \%$, and MS/MS ions were detected using the ion trap analyzer. Tandem mass spectra were screened with a suitable protein database with Mascot (Matrix Science) with "Trypsin/P" enzyme cleavage, static cysteine alkylation by chloroacetamide and variable methionine oxidation. Label-free quantitation was performed with the MaxQuant software. Protein specifications used to cluster identified proteins derived from The Universal Protein Resource (UniProt). Proteins identified from the corresponding EYFP Co-IP or biotin pull-down were considered background and excluded from data obtained from EYFPDAAO Co-IP or propiverine pull-down, respectively. All proteins that were enriched $>2$-fold in LFQ intensity compared to EYFP control were ranked as a hit in EYFP-DAAO-Co-IP analyses.

\subsection{Western blot analysis}

Samples were separated by a $12 \%$ SDS-PAGE and blotted onto a nitrocellulose membrane. After staining with Ponceau S, the membrane was blocked with $5 \%(\mathrm{w} / \mathrm{v})$ milk powder in TTBS $(100 \mathrm{mM}$ Tris- $\mathrm{HCl}$, $150 \mathrm{mM} \mathrm{NaCl}, 0.1 \%$ (v/v) Tween 20, pH 7.6) for $40 \mathrm{~min}$. The following antibodies were diluted in $5 \%$ milk powder and incubated $\mathrm{O} / \mathrm{N}$ at $4{ }^{\circ} \mathrm{C}$ : anti-GFP (1:2000, Roche, \#18144600001), anti-MFE2 (1:2000, origene, TA507028), TCP1 (1:300, Santa Cruz, sc-374088), PEX5 (1:500, Abcam, ab125689). After washing, membranes were incubated for $1 \mathrm{~h}$ at RT with peroxidase-conjugated secondary antibodies (anti-mouse IgGperoxidase, 1:80000, Sigma, \#A9044) and signals were detected by enhanced chemiluminescence (ECL ultra, Lumigen, \#TMA-6).

\subsection{Immunohistochemistry}

Archived FFPE kidney sections of 1000 ppm propiverine- and placebo-exposed male F344 rats [3] were deparaffinized, rehydrated and microwaved for $20 \mathrm{~min}$ in Tris-EDTA buffer ( $\mathrm{pH}$ 9). After blocking for $20 \mathrm{~min}$ in $1 \% \mathrm{BSA}+2 \% \mathrm{FCS}$, sections were incubated over night at $4{ }^{\circ} \mathrm{C}$ with primary antibodies listed in Table S1. After washing, sections were probed for $1 \mathrm{~h}$ at room temperature with AlexaFluor-conjugated secondary antibodies (Life Technology) and stained for $15 \mathrm{~min}$ with $0.5 \%$ Sudan Black B to reduce autofluorescence [28]. Finally, sections were counterstained with Hoechst 33342 and mounted (Dako, \#S3023). Control stainings (without primary and secondary antibodies, respectively) were performed to rule non-specific antibody reactivity. Peroxisomal localization of all tested proteins was confirmed using costaining with PMP70, a peroxisomal membrane protein (data not shown). Note that, cytosolic and nuclear protein accumulations in propiverine-exposed rat kidney tissue are visible in bright field images without immunohistochemical staining [11] and were used to localize fluorescent-labeled accumulations in matters of co-localization. Cytosolic and nuclear accumulations are indicated by white arrows and displacement of chromatin, respectively.

\subsection{Confocal microscopy}

Kidney sections were imaged using a point laser scanning confocal microscope LSM 880 (Zeiss) equipped with a $63.0 \times / 1.40$ oil immersion objective and ZEN software (Zeiss). Multiple fluorophores were imaged via sequential scanning. All pictures are representative for at least three independent experiments $(n=3)$. If not stated otherwise, the same laser intensities were applied for sections of control and propiverinetreated rats.

\subsection{Data handling and statistical analyses}

Image analysis was performed using (Fiji Is just) ImageJ software. To determine the peroxisome number per visual field (VS), PMP70 positive particles were quantified as described in Ref. [11] using (Fiji Is just) ImageJ software. Briefly, Otsu's method was used for automated clustering-based image thresholding, with a subsequent background filling of particles and watershed-segmentation. At least three representative visual fields each with four to seven tubules were randomly selected from each kidney slide. GraphPad Prism 5 was used for statistical analysis.

\section{Results}

\subsection{DAAO interaction partners in HEK293 and WKPT cells}

EYFP-tagged rat and human DAAO (hereafter referred to as EYFPrDAAO and EYFP-hDAAO, respectively), and mutants lacking the PTS1 sequence (DAAO- $\triangle$ PTS) thereof were used to identify DAAO-specific interaction partners in HEK293 and WKPT cells. As described earlier [12], EYFP-rDAAO and EYFP-hDAAO were predominantly localized in peroxisomes due to the C-terminal PTS1 sequence and are subsequently referred to as $\mathrm{DAAO}_{\text {perox }}$, while the DAAO- $\triangle \mathrm{PTS}$ mutants lacking the Cterminal PTS1 sequence were localized simultaneously in the cytosol and the nucleus [12] and are subsequently referred to as DAAO ${ }_{\text {cyt. }}$ GFPTrap coupled magnetic beads allowed efficient immobilization and purification of EYFP-DAAO (Fig. 1 A, eluate) from HEK293 cell lysates (Fig. $1 \mathrm{~A}$, input). Concomitantly Western blot analyses were carried out to confirm that comparable amounts of DAAO were used for all samples in the subsequent nano-LC-ESI-MS/MS analyses to identify potential 


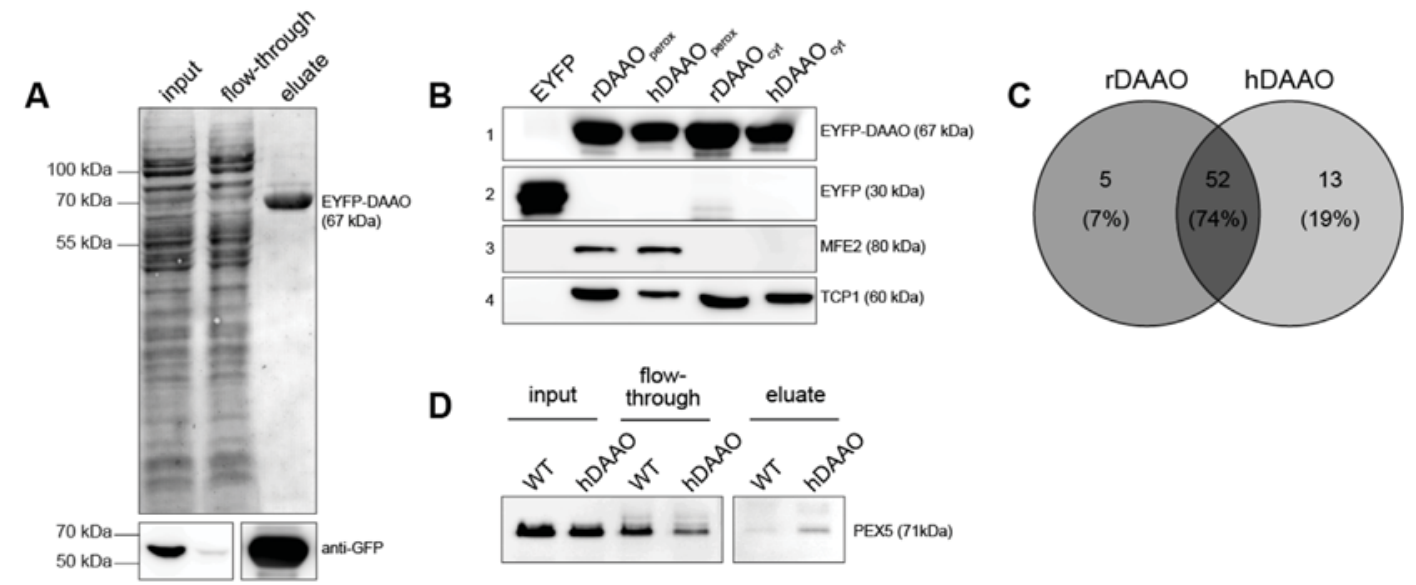

Fig. 1. Co-immunoprecipitation of DAAO interaction partners in HEK293 cells.

A) Representative Ponceau S staining (upper panel) and Western blot analysis (lower panel) of EYFP-DAAO pull-down from HEK293 cell lysate. EYFP-DAAO expressed in HEK293 cells (input) was immobilized to GFP-Trap" coupled magnetic beads and, after intensive washing (flow-through), successfully purified (eluate). Some weak bands in the eluate indicate that DAAO-binding partners were co-eluted. EYFP-DAAO (67 kDa) was detected via anti-GFP antibodies (lower panel). Note that input/flow-through and eluates were detected separately due to a highly variable protein content. B) Western blot analysis of pooled eluates from three biological replicates confirmed the efficient immobilization of EYFP and EYFP-DAAO (line $1+2$ ). The two most abundant interaction partners identified by nano-LC-ESI-MS/MS, MFE2 and TCP1, are efficiently detected in the eluates via Western blotting (line $3+4$ ). MFE2 is exclusively present in samples containing peroxisomal DAAO (DAAO perox $_{\text {, line } 3 \text { ) and TCP1 in samples with both, peroxisomal (DAAO }}$ perox $)$ and cytosolic/nuclear (DAAO cyt $_{\text {s }}$ subtypes (line 4). C) Venn diagram of identified rDAAO- and hDAAO-specific interactors in HEK293 cells sharing $74 \%$ mutual binding partners. Digits indicate the number of identified proteins in the respective cluster, a subset of which are listed in Table S4. D) Co-IP of PEX5 in HEK293 cells transiently transfected with DAAO. Co-IP was performed 15 h after transfection with nontransfected HEK293 (WT) and EYFP-hDAAO (indicated as hDAAO) expressing cells confirming the interaction of PEX5 and EYFP-hDAAO (eluate). Note that input/flow-through and eluates were detected separately due to a highly variable protein content and that PEX5 was not identified in the WT eluate via nano-LC-ESI-MS/MS.

\section{Table 1}

rDAAO interaction partners in HEK293 cells. Ten most abundant proteins identified as possible EYFP-tagged rat DAAO (EYFP-rDAAO) interaction partners in HEK293 cells using Co-IP and nano-LC-ESI-MS/MS. Proteins listed according to their abundance based on their LFQ intensity. Analyses were performed with two DAAO subtypes either localized to peroxisomes (DAAOperox; indicated as perox) or deleted in their PTS1 sequence (DAAOcyt; indicated as cyt) and therefore concomitantly localized in the cytosol and the nucleus [12].

\begin{tabular}{|c|c|c|c|}
\hline & Protein ID & Protein names & DAAO subtype \\
\hline 1 & P17987; P78371; P49368; P50991; P48643; P40227; Q99832; P50990 & T-complex protein 1 (8 subunits) & perox + cyt \\
\hline 2 & P51659 & Peroxisomal multifunctional enzyme type 2 & perox \\
\hline 3 & P04040 & Catalase & perox \\
\hline 4 & Q71UM5; P42677 & 40S ribosomal protein S27; S27-like & perox + cyt \\
\hline 5 & Q13885; Q9BVA1 & Tubulin beta-2A chain; beta-2B chain & perox + cyt \\
\hline 6 & P07197 & Neurofilament medium polypeptide & perox + cyt \\
\hline 7 & P31689 & DnaJ homolog subfamily A member 1 & perox + cyt \\
\hline 8 & P07900 & Heat shock protein HSP 90-alpha & perox + cyt \\
\hline 9 & P31943; P55795 & Heterogeneous nuclear ribonucleoprotein $\mathrm{H} ; \mathrm{H} 2$ & perox \\
\hline 10 & Q02978 & Mitochondrial 2-oxoglutarate/malate carrier protein & perox + cyt \\
\hline
\end{tabular}

Perox $=$ peroxisomal, cyt $=$ cytosolic.

DAAO interaction partners (Fig. $1 \mathrm{~B}$, lines $1+2$ ). Note that proteins identified in cells exclusively expressing EYFP (Fig. 1 B, line 2) were considered background and subtracted from hits obtained for EYFPDAAO. In total, 57 and 65 interaction partners of rDAAO and hDAAO were identified in HEK293 cells (Tables S2 and S3, Fig. 1C), respectively. The ten most abundant proteins interacting with rDAAO and hDAAO were listed in Tables 1 and 2. Notably, T-complex protein 1 (TCP1) and peroxisomal multifunctional enzyme type 2 (MFE2; Tables 1 and 2) ranked first both for rDAAO and hDAAO, as was also demonstrated by Western blot (Fig. $1 \mathrm{~B}$, lines $3+4$ ). TCP1 is a member of the chaperonin containing TCP1 complex (CCT), also known as TCP1 ring complex (TRiC). The complex consists of two rings, each containing eight different proteins $(\alpha-\theta)$ [29]. Strikingly, all eight subunits of TCP1 were identified as interaction partners of rat and human DAAO (Tables S2 and S3). TCP1 is known to be post-translationally involved in import of newly synthesized peroxisomal matrix proteins by mediating correct folding during synthesis [14]. Consequently, TCP1 should also be an interaction partner of the cytosolic/nuclear DAAO subtype $\left(\mathrm{DAAO}_{\text {cyt }}\right)$ as was confirmed by nano-LC-ESI-MS/MS (Tables 1 and 2; Tables S1, S2) and Western blot (Fig. 1 B, line 4). The second protein, MFE2, also known as D-bifunctional protein (DBP) or 17- $\beta$-hydroxysteroid dehydrogenase 4 (Hsd17b4), is a peroxisomal matrix protein and is targeted to peroxisomes via a PTS1. MFE2 contains two enzymatic domains, i.e. 2-enoyl-CoA hydratase and 3-hydroxyacyl-CoA dehydrogenase activity, and is responsible for the second and third step of fatty acid $\beta$-oxidation [30]. In contrast to TCP1, MFE2 was identified as an interaction partner of only peroxisomally localized rDAAO and hDAAO (Fig. 1 B, line 3). Similarly, catalase (CAT) - also a prominent PTS1- and peroxisomal marker protein - was identified only in samples with peroxisomal DAAO (Tables S2 and S3). The latter suggested, on the one hand, that the interaction of DAAO with MFE2 and with CAT occurred exclusively within peroxisomes, and on the other hand, that the applied Co-IP approach reflected the expected protein-protein interaction within the intracellular protein network [31]. Accordingly, it was not surprising to find tubulin, several chaperones (heat shock protein HSP90 $\alpha$, DnaJ homolog subfamily A member 1 (DnaJA1) and DnaJC7) as well as mitochondrial and nuclear proteins amongst the ten most abundant interaction partners present for both, rDAAO and hDAAO (Tables 1 and 2), especially since recent findings revealed that subcellular compartments should no longer be considered isolated entities [31]. As peroxisomes and mitochondria are actively involved in a joint lipid metabolism, it would appear evident that metabolites [32] and dual targeted enzymes [33] are exchanged. Indeed, the latter is corroborated by the fact that mitochondrial proteins, e.g. mitochondrial 


\section{Table 2}

hDAAO interaction partners in HEK293 cells. Ten most abundant proteins identified as possible EYFP-tagged human DAAO (EYFP-hDAAO) interaction partners in HEK293 cells using Co-IP and nano-LC-ESI-MS/MS. Proteins listed according to their abundance based on their LFQ intensity. Analyses were performed with two DAAO subtypes either localized to peroxisomes (DAAOperox; indicated as perox) or deleted in their PTS1 sequence (DAAOcyt; indicated as cyt) and therefore concomitantly localized in the cytosol and the nucleus [12].

\begin{tabular}{|c|c|c|c|}
\hline & Protein ID & Protein names & DAAO subtype \\
\hline 1 & $\begin{array}{l}\text { P17987; P78371; P49368; } \\
\text { P50991; P48643; P40227; } \\
\text { Q99832; P50990 }\end{array}$ & $\begin{array}{l}\text { T-complex protein } 1 \text { ( } 8 \\
\text { subunits) }\end{array}$ & perox + cyt \\
\hline 2 & P51659 & $\begin{array}{l}\text { Peroxisomal multifunctional } \\
\text { enzyme type } 2\end{array}$ & perox \\
\hline 3 & Q13885; Q9BVA1 & $\begin{array}{l}\text { Tubulin beta-2A chain; beta- } \\
2 B \text { chain }\end{array}$ & perox + cyt \\
\hline 4 & O75521 & Enoyl-CoA delta isomerase 2 & perox + cyt \\
\hline 5 & Q71UM5; P42677 & $\begin{array}{l}\text { 40S ribosomal protein S27; } \\
\text { S27-like }\end{array}$ & perox + cyt \\
\hline 6 & P07900 & $\begin{array}{l}\text { Heat shock protein HSP 90- } \\
\text { alpha }\end{array}$ & perox + cyt \\
\hline 7 & Q9Y265 & RuvB-like 1 & perox + cyt \\
\hline 8 & Q99615 & $\begin{array}{l}\text { DnaJ homolog subfamily C } \\
\text { member } 7\end{array}$ & perox + cyt \\
\hline 9 & P31943; P55795 & $\begin{array}{l}\text { Heterogeneous nuclear } \\
\text { ribonucleoprotein } \mathrm{H} ; \mathrm{H} 2\end{array}$ & perox + cyt \\
\hline 10 & P35613 & Basigin & perox + cyt \\
\hline
\end{tabular}

Perox $=$ peroxisomal, cyt $=$ cytosolic.

transporters, chaperones and enzymes involved in fatty acid $\beta$-oxidation (Tables S2 and S3), were identified as DAAO interaction partners. Overall, the majority (74\%) of identified DAAO interaction partners in HEK293 cells co-immunoprecipitated with both rDAAO and hDAAO, possibly due to their $80 \%$ sequence homology and nearly identical surface topology [25], whereas $19 \%$ were identified as hDAAO-specific and $7 \%$ as rDAAO-specific (Fig. 1C, Table S4). None of the rDAAOspecific interaction partners could be readily associated with a function that would explain a role in rDAAO mislocalization in vivo (Table S4). Similarly, of the ten hDAAO-specific interacting proteins only one, i.e. HSC70 (Table S4), could be directly involved in the regulation of DAAO trafficking [14-17]. Interesting candidates identified for both DAAO species - involved in the maintenance of cellular protein homeostasis are the E3 ubiquitin-protein ligase CHIP and several chaperones (e.g. $60 \mathrm{kDa}$ heat shock protein, heat shock protein HSP90 $\alpha$; Tables S2 and S3).

To overcome limitations of the human cell system with regard to the potentially rat-specific phenomenon [3], DAAO-specific interaction partners were identified from WKPT (WKY rat, kidney proximal tubule) cells. Although TCP1 was also identified as the most abundant DAAO interaction partner, no other rat-specific proteins could readily be associated with DAAO trafficking and protein accumulation/localization (data not shown).

An expected DAAO interaction partner that however was not found - apart from pLG72, which is exclusively present in primate neuronal cells [27] - was PEX5, i.e. the rat and human cytosolic PTS1 receptor that guides PTS1 cargos to the peroxisomal membrane [18]. Based on PEX5 function, the interaction between PEX5 and its cargo should be rather brief [13]. Indeed, a very low and transient level of PEX5-DAAO interaction is expected when considering the model system used, i.e. stably expressing EYFP-DAAO HEK293 cells where DAAO is primarily localized to peroxisomes [12]. To demonstrate the rather transient and brief interaction of DAAO with PEX5, HEK293 cells were transiently transfected with EYFP-hDAAO and a Co-IP was initiated directly after transfection. The latter approach increased the pool of cytosolic DAAO and thus PEX5-DAAO interaction (Fig. $1 \mathrm{D}$, hDAAO eluate), since peroxisomal import had not yet occurred [12,27]. Assuming that no other protein than PEX5 would specifically bind to the PTS1 motif of DAAO, the deletion of the PST1 motif will prevent PEX5 binding and thus shuttling and incorporation of DAAO into the peroxisomes [12], thereby providing sufficient amounts of cytosolic and nuclear DAAO to allow for the identification of additional DAAO interaction partners. Indeed, the latter interaction partners could be key candidates for an interaction with propiverine and/or its metabolites that then would lead to the observed nephropathy in vivo. Contrary to expectations, no particular interaction partners of DAAO- $\triangle$ PTS-mutants could be directly associated with potential dysregulation of DAAO trafficking and/or accumulation (Tables S2 and S3). In view of the fact that in the human and in the rat cell systems DAAO- $\Delta$ PTS-mutants presented with a distinct cytosolic and nuclear distribution [12] and that chaperones (TCP1, HSC70/DnaJ, HSP90, HSP60) appeared as abundant DAAO interaction partners, it was hypothesized that propiverine could bind to either PEX5 or to one or more chaperones and thus dysregulate DAAO trafficking and/or peroxisomal import. Moreover, the interaction of propiverine with PEX5 and/or chaperones could also affect other peroxisomal matrix proteins. To test these hypotheses and to better emulate the rat in vivo situation, propiverine-specific interaction partners were identified from rat kidney cortex homogenates.

\subsection{Propiverine interacts with several PTS1 proteins and enzymes involved in protein homeostasis}

To allow identification of propiverine-specific interaction partners, a biotin-labeled propiverine derivative was synthesized (Fig. 2 A): Biotin-functionalized propiverine $\mathbf{1 1}$ was synthesized starting from benzilic acid 1. After a two-step esterification via benzilic acid methyl ester 2, $N$-methyl-4-piperidyl benzilate 4 was obtained, which was converted to the corresponding chlorobenzilate 5. Subsequently, oligo (ethylene glycol) azide 6, designated as a long hydrophilic linker, was coupled to 5 to obtain ether $\mathbf{7}$. Subsequent Staudinger reduction to the corresponding amine $\mathbf{8}$ allowed coupling with D-biotin-pentafluorophenyl (Pfp) ester $\mathbf{1 0}$ which yielded biotinylated propiverine $\mathbf{1 1}$ as a white solid. The detailed description of the synthetic steps is given in the Supplementary Information.

Biotin-linked propiverine $\mathbf{1 1}$ was immobilized on streptavidincoated magnetic beads and incubated with rat cortex homogenate (Fig. $2 \mathrm{~B}$, input) to co-precipitate renal propiverine interactors. Intensive washing (flow-through) and unmodified D-biotin (Fig. 2 B) were used to break non-specific interactions and as background control, respectively, to eliminate non-propiverine-specific binding partners. Although, the resulting number and quantity of interacting proteins was too low for visual detection (Fig. 2 B, eluates), eluted proteins were identified via nano-LC-ESI-MS/MS. The propiverine-binders were listed in order of their abundance and classified, according to the elution method used (i.e. a mild (1st) elution and a harsh (2nd) elution) into potential weak and strong interactors (Table S5). A total of 80 propiverine-specific interaction partners were identified from the rat renal cortex homogenates (Table S5) and were clustered based on joint cellular pathways, protein class or sub-cellular destination in Fig. 2C and Table S6. The largest cluster of propiverine-specific interaction partners represented 26 proteins involved in the maintenance of protein homeostasis including protein biosynthesis, folding and degradation. Eight of these belong to the class of chaperones (DnaJ homolog subfamily A member 1 (DnaJA1) and 2 (DnaJA2), 70 heat shock protein (HSP701A, HSP701B), heat shock $70 \mathrm{kDa}$ protein $4,60 \mathrm{kDa}$ heat shock protein, hypoxia up-regulated protein), while six were identified as enzymes involved in protein degradation, e.g. several peptidases and two proteasomal subunits (Fig. 2C). Amongst the latter, the $26 \mathrm{~S}$ proteasome non-ATPase regulatory subunits 1 and 2 (Rpn1 and Rpn2) were part of the first 20 hits of the strong interactors (Table S5, line 19 and 29). Rpn1 and Rpn2, i.e. the non-ATPase subunits of the 19S regulator particles, are involved in substrate recognition and binding for subsequent proteolytic degradation [34]. The second largest cluster represented 18 proteins that are all members of the oxidoreductase family. Among these, the three peroxisomal enzymes identified: DAAO, 
A<smiles>O=C(O)C(O)(c1ccccc1)c1ccccc1</smiles>
$\mathrm{MeOH}_{4} \mathrm{H}_{2} \mathrm{SO}_{4}$.<smiles>COC(=O)C(O)(c1ccccc1)c1ccccc1</smiles>
$\underbrace{\overbrace{\mathrm{OH}, \mathrm{NaOMe}}^{\mathrm{N}}}_{51 \%}$

$\bigcup_{0}^{N}$

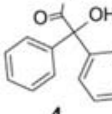

pyridine, $\mathrm{SOCl}_{2}$.<smiles>CC(C)COCCO</smiles><smiles>CN1CCC2CCCCC2C1</smiles><smiles>[Z]c1ccccc1C(OCCOC(C)CN)(c1ccccc1)C(O)(OCCC)OCCOCC</smiles>
$\underset{78 \%}{\stackrel{\mathrm{PPh}_{3}, \mathrm{H}_{2} \mathrm{O}}{\longrightarrow}}$<smiles>CN1CC=CCC1</smiles>
of of 0$)_{3} \mathrm{NH}_{2}$<smiles>c1ccc2c(c1)CCCC2</smiles>

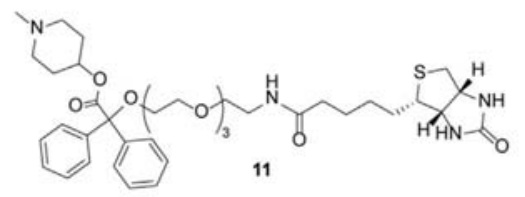

B

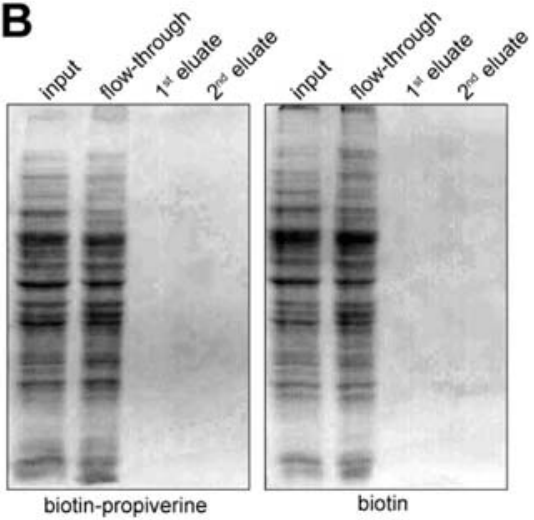<smiles>[R]OC(=O)CCCC[C@H]1SC[C@@H]2NC(=O)N[C@H]21</smiles>

C

\begin{tabular}{l}
\hline Cluster of interaction partners \\
\hline Total number of proteins \\
Protein homeostasis \\
$\quad$ chaperones \\
$\quad$ protein degradation \\
Oxidoreductases \\
Mitochondrial proteins \\
Cytoskeleton \\
Channels and transporter \\
Fatty acid metabolism \\
Metabolizing proteins and detoxification \\
Amino acid metabolism \\
Signal transduction \\
Carbohydrate metabolism \\
Vesicle-mediated transport \\
Peroxisomal proteins \\
\hline
\end{tabular}

\begin{tabular}{ll}
\hline Chaperones & \\
\hline Protein ID & Protein \\
\hline P0 DMW1;P0DMW0 & Heat shock 70 kDa protein 1B; -1A \\
O35824 & DnaJ homolog subfamily A member 2 \\
P63036 & DnaJ homolog subfamily A member 1 \\
O88600 & Heat shock 70 kDa protein 4 \\
P63039 & 60 kDa heat shock protein \\
Q66HD0 & Endoplasmin \\
P35565 & Calnexin \\
Q63617 & Hypoxia up-regulated protein 1 \\
\hline \multicolumn{2}{|c}{ Protein degradation } \\
\hline Protein ID & Protein \\
\hline P15684 & Aminopeptidase N \\
Q9EQV6 & Tripeptidyl-peptidase 1 \\
Q64230 & Meprin A subunit alpha \\
Q4FZT9 & 26S proteasome non-ATPase regulatory subunit 2 \\
O88761 & 26S proteasome non-ATPase regulatory subunit 1 \\
Q68FS4 & Cytosol aminopeptidase \\
\hline \multicolumn{2}{|c}{} \\
\hline Peroxisomal proteins \\
\hline Protein ID & Protein \\
\hline O35078 & D-amino-acid oxidase \\
P97852 & Peroxisomal multifunctional enzyme type 2 \\
Q07523 & Hydroxyacid oxidase 2 \\
\hline
\end{tabular}

D

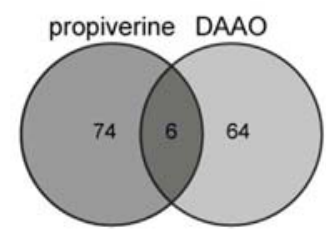

\begin{tabular}{lll}
\hline & Protein ID & Common propiverine and DAAO binding partners \\
\hline $\mathbf{1}$ & Q63716 & Peroxiredoxin-1 \\
$\mathbf{2}$ & P63036 & DnaJ homolog subfamily A member 1 \\
$\mathbf{3}$ & P51659 & Peroxisomal multifunctional enzyme type 2 \\
$\mathbf{4}$ & P10809 & 60 kDa heat shock protein \\
$\mathbf{5}$ & Q9BVA1;Q13885 & Tubulin beta-2A and 2B chain \\
$\mathbf{6}$ & P62879; Q9HAV0 & Guanine nucleotide-binding protein $\mathrm{G}(\mathrm{I}) / \mathrm{G}(\mathrm{S}) / \mathrm{G}(\mathrm{T})$ subunit beta-2; subunit beta-4 \\
\hline
\end{tabular}

Fig. 2. Identification of propiverine-specific interaction partners from rat kidney.

A) Synthesis of biotin-labeled propiverine. D-Biotin $\mathbf{9}$ was coupled to $N$-methyl-4-piperidyl benzilate $\mathbf{5}$ via a polyethylene glycol linker $\mathbf{6}$ yielding a biotin-functionalized propiverine derivative 11 (see supplementary material for details). B) Pull-down analysis of biotin-labeled propiverine. Biotinylated propiverine 11 or D-biotin 9 was immobilize on streptavidincoated magnetic beads. Rat kidney cortex homogenate (input) was added to co-precipitate specific renal interaction partners after washing (flow-through) to remove non-specific binders. A mild high salt (1st eluate) and harsh elution, i.e. EDTA-formamide at $90^{\circ} \mathrm{C}$ (2nd eluate), were performed considering the identified proteins as weak or strong interactors, respectively (see Table S5). C) Cluster of propiverine interaction partners from rat kidney. Proteins identified from propiverine pull-down analysis were clustered according to joint cellular pathways, protein class or sub-cellular destination (for full list of clustered interaction partners see Table S6). D) Mutual binding partners of propiverine and DAAO. Six DAAO-specific (either rat and/or human DAAO) interaction partners were identified in propiverine pull-down analysis from rat kidney homogenate.

hydroxyacid oxidase 2 (HAOX2) and MFE2, contain all a PTS1 motif, i.e. their peroxisomal import depends on PEX5. HAOX2 and MFE2, are involved in fatty acid metabolism [30,35].

Confidence in the methodological approach employed was gained from the fact that enzymes involved in the biotransformation of xenobiotics and drugs were identified as propiverine-specific interaction partners from the rat renal cortex (Table S5) and liver (Table S8) homogenates, especially since propiverine was found to be highly metabolized in liver and contributed to specific hepatic and renal metabolites [36,37]. Moreover, propiverine was also shown to bind to guanine nucleotide-binding proteins (G-proteins), as would be expected from the fact that G-proteins are functionally and locally tightly associated with the muscarinic receptor of the bladder and the kidney $[38,39]$ and thus an intricate factor of the anti-muscarinic function of propiverine in the treatment of symptoms associated with overactive bladder syndrome [6]. Confidence in the proteins identified was further supported by the fact that the Co-IP analyses from liver homogenates (Table S7) corroborated the findings obtained from kidney samples (Fig. 2C). The majority of propiverine-specific binders from the liver samples represented proteins involved in protein homeostasis and enzymes belonging to the oxidoreductase family, including several peroxisomal enzymes (Tables S7 and S8). 
The comparison of DAAO- and propiverine-specific interaction partners provided for six proteins in the rat kidney models (Fig. $2 \mathrm{D}$ ): MFE2, peroxiredoxin-1, DnaJA1, $60 \mathrm{kDa}$ heat shock protein, tubulin $\beta 2 \mathrm{~A} / \mathrm{B}$ and guanine nucleotide-binding protein (G-protein) subunit $\beta 2$ and $\beta 4$. Peroxiredoxin- 1 is an antioxidant enzyme, i.e. a member of the oxidoreductase family, generally found in the cytosol and nucleus where it effectively detoxifies $\mathrm{H}_{2} \mathrm{O}_{2}$ [40]. DnaJA1 belongs to the DnaJfamily of Hsp40 co-chaperones and belongs to the four major Hsp40 chaperones present in the cytosol [41], while tubulin $\beta 2 \mathrm{~A} / \mathrm{B}$ is a structural protein and guanine nucleotide-binding protein subunit $\beta 2$ and $\beta 4$ are part of the heterotrimetic G-proteins involved as modulators or transducers in various transmembrane signaling systems, e.g. muscarinic receptor-mediated bladder contraction [42].

Based on above findings that propiverine appeared to interact with several other PTS1 proteins, beyond DAAO, it appeared most likely that propiverine would affect protein trafficking at a much broader level than previously assumed. Consequently, several peroxisomal proteins, as well as typical chaperones and PEX proteins involved in peroxisomal trafficking and homeostasis were analyzed within kidney sections from control and propiverine-treated rats using immunofluorescence and confocal microscopy.

\subsection{Mislocalization of PTS1 and selected PEX proteins but not of PTS2 proteins and PMPs after propiverine exposure}

Of the key proteins involved in protein folding, chaperon-mediated shuttling and peroxisomal import, TCP1, the protein responsible for folding of peroxisomal matrix proteins and main DAAO in vitro interactor, did not present with a different intracellular distribution nor accumulation following propiverine treatment (Fig. 3 A). However, confocal analysis of HSC70 and the HSP40 co-chaperon DnaJB1, involved in chaperon-mediated shuttling and peroxisomal import $[14,15]$, demonstrated that HSC70 accumulated in the nuclei (surrounded by displaced chromatin) and cytosol (white arrows) of propiverine-treated rats (Fig. 3 B), while DnaJB1 accumulated primarily in the nuclei (Fig. 3C). In contrast, HSP90, not involved in mammalian peroxisomal protein trafficking $[14,15]$, presented no altered cytosolic or nuclear distribution when compared to control animals (Fig. $3 \mathrm{D}$ ) and thus strongly supported the previous assumption that propiverine interferes with peroxisomal protein transport. PEX5, responsible for the import of PTS1 proteins into peroxisomes, accumulated primarily in the cytosol (Fig. 3 E). This specific cytosolic localization and the fact that HSC70 and DnaJB1 and the PTS1 receptor PEX5 co-localize with their cargo protein, i.e. DAAO (Fig. $3 \mathrm{~F}$ ) can be interpreted as the result of two processes: a) direct interaction of propiverine with either PTS1proteins or proteins involved in PTS1 protein shuttling (e.g. HSC70), but not with PEX5 (Table S5), resulting in the formation of propverinePTS1-HSC70/HSP40 chaperone complexes with or without PEX5, and b) active export of PEX5 and PEX5-bound cargo from the nucleus due to the presence of a CRM1 binding motif [43], as will be discussed below.

The observation that HSC70 and PEX5, both essential for peroxisomal transport/import were affected after propiverine exposure, suggests, as presumed earlier, that other PTS1 proteins should be affected as well. Indeed, the confocal analyses of MFE2, acyl-coenzyme A oxidase 1 (ACOX1), CAT and enoyl-CoA hydratase/3-hydroxyacyl CoA dehydrogenase (EHHADH), demonstrated a propiverine-dependent accumulation and/or mislocalization (Fig. 3G-J). All of the mentioned PTS1 proteins, except CAT, are involved in peroxisomal fatty acid $\beta$ oxidation. ACOX1 catalyzes the first [44] and EHHADH, similar to MFE2, the second and third step of $\beta$-oxidation while possessing both hydratase and dehydrogenase activity, wherefore it is also known as Lbifunctional enzyme (L-PBE) [45]. However, while MFE2 and ACOX1 presented with a nuclear and cytosolic accumulation (Fig. 3G-H), comparable to that observed for DAAO (Fig. 3 F), CAT and EHHADH presented with a cytosolic and nuclear diffuse mislocalization lacking distinct accumulations (Fig. 3I-J). The latter could be due to size restrictions of the nuclear pore complex hindering diffusion into the nucleus, e.g. in the case of CAT [12], and/or differences in protein solubility under physiological conditions. Irrespective of the latter, the presented data strongly suggest that the complete PTS1 matrix protein trafficking pathway is affected by propiverine. Indeed, the analysis of the mammalian PTS2 proteins acetyl-coenzyme A acyltransferase 1 (ACAA1) [15], catalyzing the last step of fatty acid $\beta$-oxidation within peroxisomes [46], and alkyl-dihydroxyacetone phosphate synthase (AGPS) [15], involved in plasmalogen (ether phospholipid) biosynthesis [47], demonstrated no changes in intracellular distribution upon propiverine treatment (Fig. 3K-L). Thus PTS2 proteins - in contrast to PTS1 proteins - appeared not affected by propiverine. Contrary to expectations, PEX7 demonstrated a diffuse cytosolic and ring-like nuclear mislocalization pattern (Fig. $3 \mathrm{M}$ ), despite that its cargoes, ACAA1 and AGPS were unaffected (Fig. 3K-L). The latter could be explained by the fact that PEX7 and PEX5L are part of the import complex for PTS2 proteins [20]. Binding of PEX7 to the PEX5L-PTS1 complex would result in the mislocalization of PEX7 as well yet without detectable consequences for PTS2 proteins (Fig. 3K-L). The latter strongly supports a specific interaction of propiverine with the PTS1-trafficking pathway and consequently suggests that peroxisomal membrane proteins (PMPs) should remain unaffected as well. Indeed, the corresponding analysis of peroxisomal membrane proteins e.g. PMP70 (also known as ATPbinding cassette subfamily D member 3 (ABCD3)), the PMP-shuttle PEX19 and the PMP incorporation machinery proteins e.g. PEX16, did not show any propiverine-induced mislocalization (Fig. 3N-P). Note that PMP70-stained peroxisomes in pl cebo animals manifested a characteristic ring-like structure indicating a specific membrane localization (Fig. $3 \mathrm{~N}$, bottom). In contrast, peroxisomes after propiverine treatment presented as small, dot-like structures due to depletion of matrix proteins (Fig. $3 \mathrm{~N}$, top), described also previously [11] and known as "peroxisomal remnants (ghosts)" in peroxisome biogenesis disorders (PBDs) [48,49]. Peroxisomal ghosts are observed only in conjunction with functional PEX3, -16 and -19 [48]. However, contrary to expectations, PEX3 accumulated and was mislocalized to the cytosol and the nucleus (Fig. 3 Q). Continuous mislocalization of PTS1 proteins, as shown above, should lead to a reduced size of the peroxisomes, as was demonstrated previously [11], and to reduced functionality. To compensate for the latter and to avoid pathological changes within the cell, a feed-back loop via increased expression of PEX3 would ensure propagated peroxisome synthesis, as PEX3 is intricately involved in the de novo biogenesis of peroxisomes [21]. Consequently an increased number of peroxisomes should be observable following propiverine exposure. Indeed, propiverine treatment resulted in a two-fold increase in peroxisome numbers, when compared to controls (Fig. 3 R).

\section{Discussion}

The analysis of propiverine interaction partners revealed an unexpected diversity of functional protein classes, i.e. chaperones, proteasomal subunits, and peroxisomal proteins (Fig. 2C and Table S5), and therefore could provide for several targets, whose dysregulation could lead to the observed propiverine-mediated nephropathy.

Propiverine binding to HSC(P)70 and DnaJ proteins could interfere with the shuttling and import of peroxisomal matrix proteins [14-17], thus one would expect that all PTS1 and PTS2 proteins would be mislocalized. However, contrary to expectations, primarily PTS1 and PEX proteins but not PTS2 proteins were affected, thus suggesting that inference with chaperones would most likely not explain the protein mislocalization observed. In addition, propiverine was found to interact with several subunits of the $26 \mathrm{~S}$ proteasome: non-ATPase regulatory subunits 1 and 2 (Rpn1 and Rpn2) in liver and kidney, involved in substrate recognition and binding [34], and the AAA-ATPases regulatory subunits 7, 4 and 8 (Rpt1, Rpt2 and Rpt6) in liver, involved in the unfolding and translocation of ubiquitylated substrates [50], 

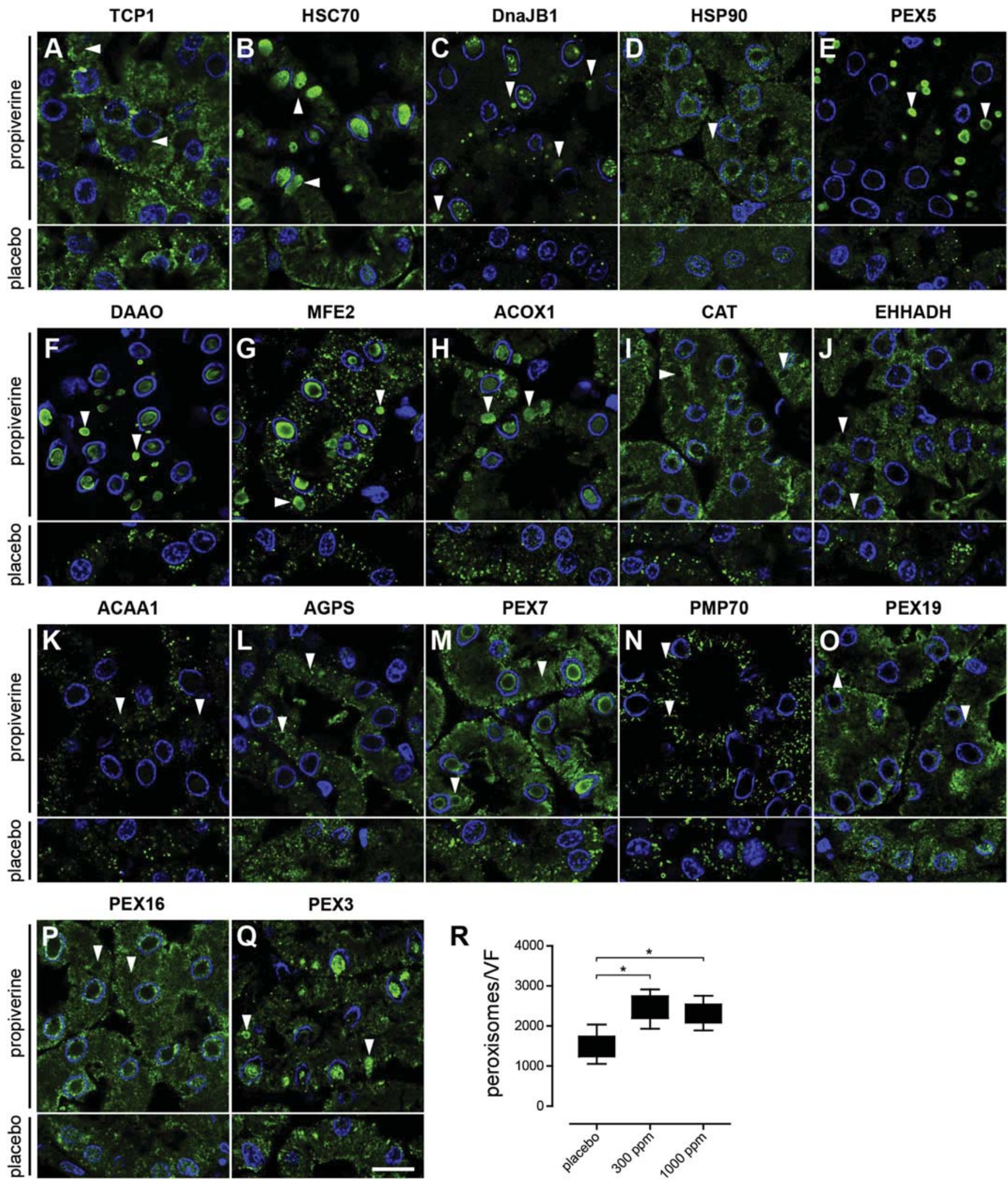

Fig. 3. Mislocalization and/or accumulation of specific peroxisomal proteins in rat kidney after propiverine exposure.

Confocal microscopy of chaperones (A-D), PTS1 matrix proteins (F-J), PTS2 matrix proteins (K-L), PMPs (N) and peroxins (E, O-Q) in rat kidney sections. Co-stainings of the proteins of interest (green) and nuclei (blue) in propiverine- (top) and placebo- (bottom) treated rats. Note that chromatin is displaced in propiverine-treated rats resulting in a ring-like appearance of nuclei due to massive protein accumulations within the nuclei. Arrows indicate localization of cytosolic protein accumulations obtained from bright-field images. Note that all accumulating proteins are colocalizing within the same accumulation entity. Scale $=15 \mu \mathrm{m}$. R) Peroxisome abundance in rat proximal tubule epithelial cells. F344 rats treated with either 300 or $1000 \mathrm{ppm}$ propiverine feature a higher number of peroxisomes in proximal tubule epithelial cells compared to placebo-treated animals. Box and Whiskers plot (min to max) show mean number of peroxisomes per visual field $(\mathrm{VF}) \pm$ SEM. One-way ANOVA with Bonferroni's post-test with *p $<.0001$. 
thereby possibly contributing to impaired proteasomal degradation. However, dose-response analyses of renal protein accumulations of propiverine-treated rats demonstrated that all renal proximal tubule cell nuclei were affected independent of dose, whereas the cytosolic accumulations increased dose-dependently [11], thereby not supporting a general inhibition of proteasomal protein degradation by propiverine. Indeed, neither the expression of proteasomal proteins nor the localization of the $26 \mathrm{~S}$ proteasome were affected by propiverine treatment in vivo [11]. Rather than provoking a complete proteasomal inhibition, propiverine may change substrate specificity or binding affinity to the Rpn1 and Rpn2 regulatory subunits in vivo which would explain the lack of an inhibitory effect of propiverine and its major metabolite in a cell-free proteasome activity assay with rat and human $20 \mathrm{~S}$ proteasomes [11]. Proteasome substrates are either directly targeted or delivered via bivalent shuttle proteins to the proteasome, i.e. the 19S regulatory particle. The $19 \mathrm{~S}$ proteasomal subunits Rpn1 and Rpn2 possess an explicit preference for the latter and recruit proteasomal targets via shuttle proteins [34]. Since different shuttle proteins not only dock at different receptor sites within one Rpn subunit but also manifest substrate selectivity [34], propiverine may be able to regulate proteasomal degradation of a specific subset of proteins e.g. PTS1 proteins, resulting in accumulation of the latter. Moreover, deletion of either Rpn subunit is lethal and mutations thereof result in impaired proteasome function, accumulation of polyubiquitinated proteins, and improper nuclear proteasome localization in yeast [34], highlighting the importance of these regulatory subunits in protein homeostasis. However, regarding the overt cell pathology associated with the observed nephropathy $[3,7,8]$, propiverine treatment most likely does not lead to a broad and strong interference of proteasomal degradation.

In view of the fact that propiverine interacted with at least three PTS1 proteins (DAAO, MFE2 and HAOX2) in rat kidney cortex homogenate, it appeared likely that propiverine treatment generally affected shuttling and import of PTS1 proteins. Indeed, the immunofluorescent analyses demonstrated that several other PTS1 proteins (MFE2, ACOX1, CAT, EHHADH) accumulated in renal cells (Fig. 3), thus contradicting previous assumptions that propiverine-mediated mislocalization was specifically restricted to DAAO [3]. In contrast, none of the PTS2 proteins or PMPs were mislocalized (Fig. 3), thereby further supporting the hypothesis that propiverine primarily dysregulates PTS1 protein trafficking. Since all of the affected PTS1 proteins in rat kidney are part of the oxidoreductase family and $>20 \%$ of all propiverine interaction partners in both kidney and liver are members of this protein class, it appeared likely that propiverine specifically binds to an oxidoreductase-specific motif. A direct interaction of propiverine with PTS1 proteins could influence their protein structure and/or interfere with proper binding of chaperones, peroxisomal receptors and/or the peroxisomal importomer, thus disturbing peroxisomal trafficking and import. Since PTS2 proteins remained unaffected, but share the same docking/translocation machinery at the peroxisomal membrane [51], the inference of propiverine with PTS1 protein transport in the cytosol appeared the most plausible mechanism of interaction.

DAAO was identified as one of the most abundant propiverine interaction partners isolated from rat kidney (Table S5) and was presumed to be the most abundant protein in rat renal accumulations [3]. Thus, DAAO could be assumed to be the primary target of propiverine interaction, despite that previous experiments suggested that neither propiverine nor its main hepatic metabolite directly interact with DAAO [11]. Indeed, these previous in situ direct-binding studies using purified DAAO may have lacked essential cofactors for efficient smallmolecule-protein interaction. The observation that other accumulating PTS1 proteins were still partially present in peroxisomes (Fig. 3), thereby maintaining their physiologically normal localization, supported DAAO as primary target of propiverine interaction. The latter would also suggest that the mislocalization of DAAO is the primary event and that the mislocalization of the other PTS1 proteins is a secondary effect. Indeed, deletion of one single peroxisomal PTS1 protein, i.e. ACOX1, was shown to be sufficient to induce mislocalization of several PTS1 proteins in ACOX1 ${ }^{-/-}$mice [52].

Under physiological conditions, peroxisomal proteins are imported in a folded and oligomeric state [14]. Thus, at first sight it appeared likely that (partially) un- or misfolded PTS1 proteins or peroxisomal proteins lacking HSC(P)70 and/or PEX5 binding [12] could remain cytosolic. However, most peroxisomal proteins are unstable when localized in the cytosol and are thus rapidly degraded [24]. The fact that propiverine-mediated nuclear protein accumulations appeared to occur earlier than cytosolic accumulations [11], could suggest that cytosolic PTS1 proteins are most likely transported into the nucleus rather than remaining in the cytosol. Indeed, as was suggested recently, DAAO is mainly degraded via the nuclear proteasome [12] and can enter the nucleus via passive diffusion [12]. Moreover, DAAO could be actively transported into the nucleus by DnaJB1 and could thus be specifically delivered for nuclear proteasomal degradation [53]. The latter assumption was supported by the fact that DnaJB1 also accumulated and co-localized with DAAO primarily in the nucleus in kidneys of propiverine-exposed rats (Fig. 3). While passive diffusion appeared to be governed by a size restriction of the nuclear pore, e.g. lack of passive import of CAT [12], active transport mechanisms allow the import of much larger molecules and protein complexes [54]. Thus the question would need to be answered whether DAAO and other PTS1 proteins reach the nucleus for proteasomal degradation as a complex with the associated chaperones (HSC70, DnaJB1) and/or PEX5 or not. The biogenesis of PTS1 proteins and their localization to the peroxisome involves ribosomal synthesis, chaperone-mediated stabilization and folding and PEX5/HSC(P)70/Hsp40-mediated transport and import into peroxisomes [13-17]. Due to the fact that the PTS1 motif is localized at the far C-terminal end of PTS1 proteins [55], PEX5 binding occurs at the very last step of PTS1 protein synthesis. Thus, propiverinebinding to PTS1-proteins, specifically to DAAO, could render two forms of misfolded PTS1-protein complexes, with and without PEX5. Assuming that misfolded PTS1-protein complexes are actively transported into the nucleus for proteasomal degradation, e.g. by DnaJB1, both forms would be present (Fig. 4). As PEX5 contains a CRM1 binding motif [43], PEX5-PTS1 complexes could be actively shuttled from the nucleus into the cytosol (Fig. 4). The latter is supported by the fact that PEX5 exclusively co-localized with cytosolic DAAO accumulations (Fig. 3). However, if the PTS1-complex lacks PEX5, it would be trapped due to the reduced mobility within the highly heterogenic nuclear environment [56] and thus would remain in the nucleus for proteasomal degradation.

Direct interference of propiverine with PEX5 functionality appeared unlikely as no propiverine binding was observed in the Co-IP studies (Table S5). Moreover, as a mutation of PEX5 located in the region necessary for PTS2 transport, e.g. PEX7 binding box, was demonstrated to affect both PTS1 and PTS2 matrix protein import in human cells [57], a non-functional PEX5 was expected to render an abnormal PTS2 protein distribution, which however was not the case (Fig. 3).

As DnaJB1, DAAO, MFE2, ACOX1 and HSC70 accumulated and colocalized in the nucleus (Fig. 3), these complexes did not appear to be readily degraded by the nuclear proteasome. A possible explanation for the latter would be that propiverine binding to DAAO renders the protein complex non-degradable. A likely reduction in the overall capacity of cellular protein homeostasis [58] due to the observed binding of propiverine to diverse chaperones, proteases and the proteasomal degradation machinery (Fig. 2 Table S5) may enhance the protein accumulation.

The finding that PEX3 accumulated following propiverine treatment would at first sight appear contradictory to above interpretation. However, in view of the fact that many of PTS1 proteins are mislocalized and only partially present in peroxisomes, it appeared likely that propiverine treatment rendered the existing peroxisomes partially non-functional. Indeed, propiverine-treated rats presented with dilatations of the hepatic ER, lipid droplets in hepatocytes and increased liver 


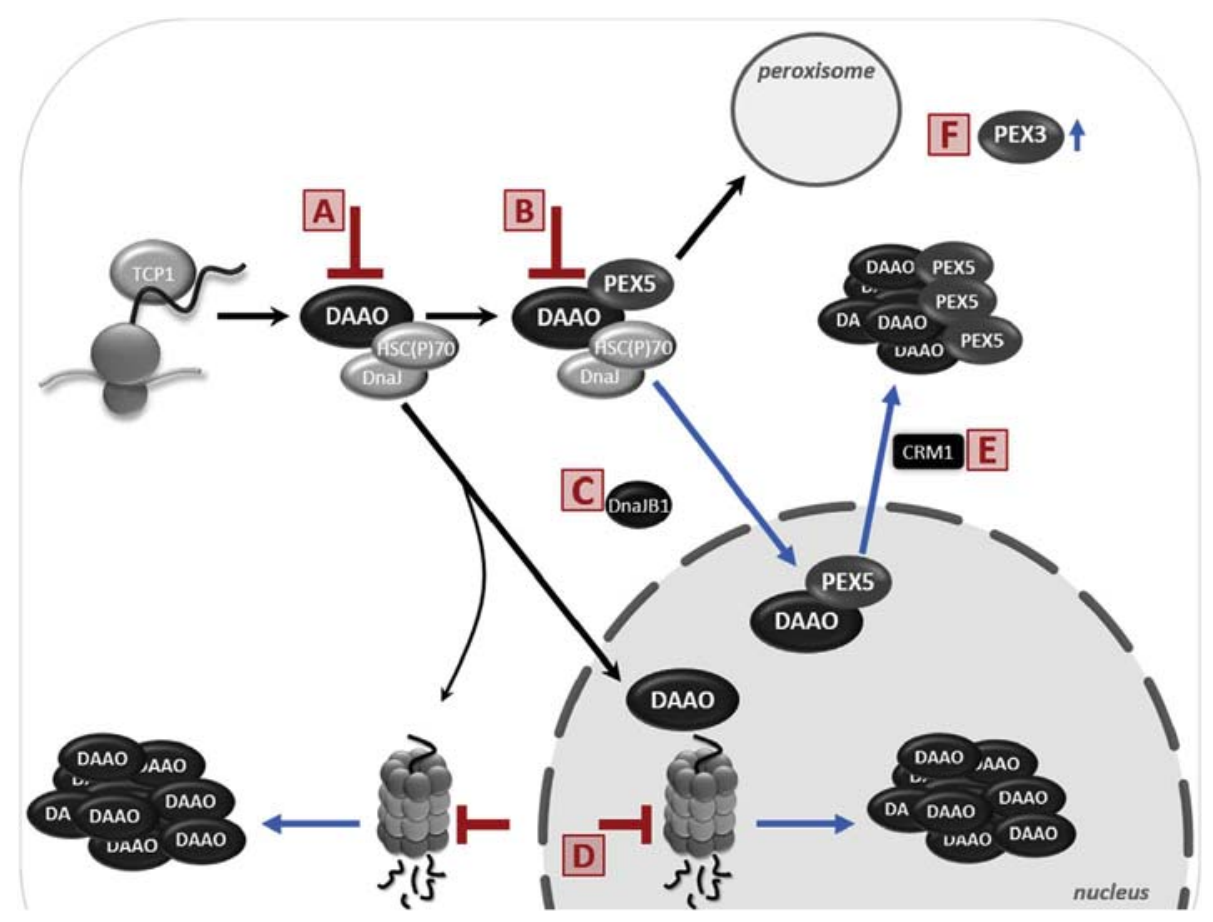

Fig. 4. Proposed mechanism of propiverine's dual effect on localization and degradation of PTS1 proteins in proximal tubule epithelial cells.

Propiverine may directly interact with DAAO and other PTS1 proteins before (A) or after (B) PEX5 binding. Direct binding of propiverine to DAAO may change its protein structure and/or abrogate chaperone (HSC(P)70, HSP40) and/or PEX5 binding, both mediating peroxisomal trafficking [14-18]. Cytosolic (mis)localized peroxisomal proteins could then enter the nucleus via passive diffusion [12] or active transport via the HSP40 chaperone DnaJB1 [53] (C). An interference of propiverine with the proteasomal degradation machinery, i.e. changing the proteasomal substrate specificity and/or binding, could provoke accumulation of mislocalized nuclear and cytosolic peroxisomal proteins (D). Nuclear PEX5-PTS1 complexes could actively be exported due to the PEX5 inherent CRM1 motif [43] resulting in the exclusive cytosolic accumulations of PEX5 (E). As a consequence of the mislocalized PTS1 proteins and thus to compensate for suboptimal functioning of peroxisomes, several PEX proteins, e.g. PEX3, are upregulated resulting in an increased peroxisome abundance (F). Black arrows $=$ physiological pathways, red $=$ propiverine sites of interaction, blue $=$ downstream effects of propiverine interaction.

weights $[7,8]$ indicative for a disturbed budding of pre-peroxisomal vesicles from the ER and an impaired lipid metabolism. PTS1 depletion due to import-incompetent peroxisomes was reported to be associated with non-functional peroxisomes, e.g. impaired peroxisome-specific metabolic pathways such as $\beta$-oxidation of fatty acids [24], which frequently results in impaired liver function (e.g. hepatomegaly) concomitant with impaired physical development [59]. PEX5 and PEX3 levels may have been increased to compensate for the lack of correctly imported PTS1 proteins. As PEX3 is intricately involved in the de novo biogenesis and degradation of peroxisomes and thus in coordinating peroxisome size and number [21], it is not surprising that an increase of peroxisome numbers (Fig. 3) as well as PPAR $\alpha$ and PPAR $\gamma$ expression levels [60] were observed after propiverine treatment. Finally, the correct incorporation of PMP70 (Fig. 3) into peroxisomes [48,61] and the lack of binding of PEX3 to propiverine (Table S5) was considered additional testimony of a functional PEX3 and thus supportive of the proposed compensatory mechanism. The observation that PEX3 is found in PTS1-positive protein inclusions in the nucleus and cytosol could be explained by the massive upregulation of PEX3 and an unspecific sequestration of freely available PEX3 into pre-existing PTS1 protein accumulations. Such protein sequestrations and compartmentalization were previously discussed as being cytoprotective or reflecting an unspecific cellular trait when dealing with protein overload [62]. The latter process was found to be reversible when the cell has regained control of normal protein homeostasis [62], as was also demonstrated for the propiverine-induced protein accumulations $[7,8]$.

As hDAAO and rDAAO interacting proteins were similar, the question arises why the propiverine-mediated misregulation of DAAO and other PTS1 proteins is still considered a rat-specific phenomenon. Indeed, preliminary analyses of renal proximal tubule epithelial cells obtained via abdominal palpation from propiverine-treated patients did not demonstrate abnormal protein inclusions or accumulations (Dietrich et al. 2008, unpublished data). Moreover, a misregulation of DAAO and other PTS1 proteins was also not observed in propiverinetreated mice or dogs [63-65].

In conclusion, the data presented suggest that propiverine - and/or a rat-specific propiverine metabolite formed in the kidney - interacts exclusively with DAAO and/or with a selected number of PTS1 proteins. The consequence of this interaction is the abrogated trafficking and peroxisomal import of PTS1 proteins concomitant with their nuclear and cytosolic accumulation due to inhibited degradation.

\section{Authors contributions}

L. Luks and M. Y. Maier planned the project, designed the experiments, performed all experiments, analyzed, interpreted and visualized all data, wrote the original draft and reviewed the manuscript. M. Y. Maier and O. Baudendistel planned the synthesis of biotin-coupled propiverine with input from V. Wittmann. M. Y. Maier performed the synthesis and planned the biotin-pull-down experiments. D. R. Dietrich provided resources, planned the project and was involved in writing, reviewing and editing the manuscript. V. Wittmann provided resources for chemical synthesis.

\section{Conflicts of interest}

The authors declare that they have no conflict of interest. This research did not receive any specific grant from funding agencies in the public, commercial or not-for-profit sectors.

\section{Ethical standards}

The manuscript does not contain clinical studies or patient data.

\section{Acknowledgements}

This work was supported by the University of Konstanz, the Konstanz Research School Chemical Biology (KoRS-CB) and the Deutsche Forschungsgemeinschaft (RTG1331). We thank the Bioimaging Center, University of Konstanz, for use of and help with the confocal microscope and the Proteomics Core Facility, University of Konstanz, for sample preparation and mass spectrometry. We acknowledge Prof. Dr. Loredano Pollegioni (University of Insubria, Italy) for providing pEYFP-C3, pEYFP-rDAAO-C3 and pEYFP-hDAAO-C3 expression vectors and Prof. Dr. Frank Thevenod (University of Witten/ Herdecke, Germany) for kindly providing WKPT cells. 


\section{Transparency document}

Transparency document related to this article can be found online at http://dx.doi.org/10.1016/j.cbi.2017.12.023.

\section{Appendix A. Supplementary data}

Supplementary data related to this article can be found at http://dx. doi.org/10.1016/j.cbi.2017.12.023.

\section{References}

[1] C. Gopinath, V. Mowat, Atlas of Toxicological Pathology, Springer, 2014.

[2] G.C. Hard, C.H. Alden, R.H. Brunder, C.H. Frith, R.M. Lewis, R.A. Owen, K. Krieg, B. Durchfeld-Meyer, Non-proliferative lesions of the kidney and lower urinary tract in rats, URG-1, Guides for Toxicologic Pathology Washington DC, 1999 STP/ARP/ AFIP; 1995.

[3] D.R. Dietrich, A.H. Heussner, E. O'Brien, T. Gramatté, M. Runkel, S. Rumpf, B.W. Day, Propiverine-induced accumulation of nuclear and cytosolic protein in F344 rat kidneys: isolation and identification of the accumulating protein, Toxicol. Appl. Pharmacol. 233 (2008) 411-419.

[4] Z.A. Radi, Z.S. Stewart, F.A. Grzemski, W.F. Bobrowski, Renal pathophysiologic role of cortical tubular inclusion bodies, Toxicol. Pathol. 41 (2013) 32-37.

[5] C. Gopinath, D.E. Prentice, D.J. Lewis, Atlas of Experimental Toxicological Pathology, Springer, 1987.

[6] K. McKeage, Propiverine: a review of its use in the treatment of adults and children with overactive bladder associated with idiopathic or neurogenic detrusor overactivity, and in men with lower urinary tract symptoms, Clin. Drug Invest. 33 (2013) 71-91.

[7] S. Nakano, M. Kuwata, H. Hasegawa, K. Irimura, A. Maruden, K. Morita, Thirteenweek oral toxicity study of propiverine hydrochloride in rats, J. Toxicol. Sci. 14 (Suppl 2) (1989) 13-59.

[8] K. Yamashita, M. Kuwata, K. Irimura, N. Morinaga, K. Kurokawa, M. Ashizawa, Fifty-two-weeks oral chronic toxicity study of propiverine hydrochloride in rats, J. Toxicol. Sci. 15 (1990) 107-144.

[9] H.A. Krebs, Metabolism of amino-acids: deamination of amino-acids, Biochem. J. 29 (1935) 1620-1644.

[10] J. Ast, A.C. Stiebler, J. Freitag, M. Bolker, Dual targeting of peroxisomal proteins, Front. Physiol. 4 (2013) 297.

[11] L. Luks, S. Sacchi, L. Pollegioni, D.R. Dietrich, Novel insights into renal d-amino acid oxidase accumulation: propiverine changes DAAO localization and peroxisomal size in vivo, Arch. Toxicol. 91 (2017) 427-437.

[12] L. Luks, M.Y. Maier, S. Sacchi, L. Pollegioni, D.R. Dietrich, Understanding renal nuclear protein accumulation: an in vitro approach to explain an in vivo phenomenon, Arch Toxicol. 91 (11) (2017 Nov) 3599-3611, http://dx.doi.org/10. 1007/s00204-017-1970-5 Epub 2017 Apr 27.

[13] P.B. Lazarow, Y. Fujiki, Biogenesis of peroxisomes, Annu. Rev. Cell Biol. 1 (1985) 489-530.

[14] V.I. Titorenko, R.A. Rachubinski, The life cycle of the peroxisome, Nat. Rev. Mol. Cell Biol. 2 (2001) 357-368.

[15] J.E. Legakis, S.R. Terlecky, PTS2 protein import into mammalian peroxisomes, Traffic 2 (2001) 252-260.

[16] C.B. Brocard, C. Jedeszko, H.C. Song, S.R. Terlecky, P.A. Walton, Protein structure and import into the peroxisomal matrix, Traffic 4 (2003) 74-82.

[17] P.A. Walton, M. Wendland, S. Subramani, R.A. Rachubinski, W.J. Welchll, Involvement of 70-kD heat-shock proteins in peroxisomal import, J. Cell Biol. 125 (1994) 1037-1046.

[18] S. Subramani, Protein import into peroxisomes and biogenesis of the organelle, Annu. Rev. Cell Biol. 9 (1993) 445-478.

[19] M.O. Freitas, T. Francisco, T.A. Rodrigues, C. Lismont, P. Domingues, M.P. Pinto, C.P. Grou, M. Fransen, J.E. Azevedo, The peroxisomal protein import machinery displays a preference for monomeric substrates, Open Biol 5 (2015).

[20] N. Braverman, G. Dodt, S.J. Gould, D. Valle, An isoform of pex5p, the human PTS1 receptor, is required for the import of PTS2 proteins into peroxisomes, Hum. Mol. Genet. 7 (1998) 1195-1205.

[21] J.J. Smith, J.D. Aitchison, Peroxisomes take shape, Nat. Rev. Mol. Cell Biol. 14 (2013) 803-817.

[22] A. van der Zand, I. Braakman, H.F. Tabak, Peroxisomal membrane proteins insert into the endoplasmic reticulum, Mol. Biol. Cell 21 (2010) 2057-2065.

[23] S.K. Lam, N. Yoda, R. Schekman, A vesicle carrier that mediates peroxisome protein traffic from the endoplasmic reticulum, Proc. Natl. Acad. Sci. Unit. States Am. 107 (2010) 21523-21528.

[24] H.R. Waterham, M.S. Ebberink, Genetics and molecular basis of human peroxisome biogenesis disorders, Biochim. Biophys. Acta (BBA) - Mol. Basis Dis. 1822 (2012) $1430-1441$

[25] L.F. Frattini, L. Piubelli, S. Sacchi, G. Molla, L. Pollegioni, Is rat an appropriate animal model to study the involvement of D-serine catabolism in schizophrenia? insights from characterization of D-amino acid oxidase, FEBS J. 278 (2011) 4362-4373.

[26] S. Sacchi, M. Bernasconi, M. Martineau, J.-P. Mothet, M. Ruzzene, M.S. Pilone, L. Pollegioni, G. Molla, pLG72 modulates intracellular D-serine levels through it interaction with D-amino acid oxidase: effect on schizophrenia susceptibility, J.
Biol. Chem. 283 (2008) 22244-22256.

27] S. Sacchi, P. Cappelletti, S. Giovannardi, L. Pollegioni, Evidence for the interaction of d-amino acid oxidase with pLG72 in a glial cell line, Mol. Cell. Neurosci. 48 (2011) 20-28.

[28] Y. Sun, H. Yu, D. Zheng, Q. Cao, Y. Wang, D. Harris, Y. Wang, Sudan black B reduces autofluorescence in murine renal tissue, Arch. Pathol. Lab Med. 135 (2011) $1335-1342$.

[29] Y. Cong, M.L. Baker, J. Jakana, D. Woolford, E.J. Miller, S. Reissmann, R.N. Kumar, A.M. Redding-Johanson, T.S. Batth, A. Mukhopadhyay, S.J. Ludtke, J. Frydman, W. Chiu, 4.0-A resolution cryo-EM structure of the mammalian chaperonin TRiC/ CCT reveals its unique subunit arrangement, Proc. Natl. Acad. Sci. Unit. States Am. 107 (2010) 4967-4972.

[30] Y. Poirier, V.D. Antonenkov, T. Glumoff, J.K. Hiltunen, Peroxisomal $\beta$-oxidation-a metabolic pathway with multiple functions, Biochim. Biophys. Acta Mol. Cell Res. 1763 (2006) 1413-1426.

[31] A.M. Valm, S. Cohen, W.R. Legant, J. Melunis, U. Hershberg, E. Wait, A.R. Cohen, M.W. Davidson, E. Betzig, J. Lippincott-Schwartz, Applying systems-level spectral imaging and analysis to reveal the organelle interactome, Nature 546 (2017) $162-167$.

[32] M. Schrader, L.F. Godinho, J.L. Costello, M. Islinger, The different facets of organelle interplay - an overview of organelle interactions, Front Cell Dev. Biol. 3 (2015) 56.

[33] O. Yogev, O. Pines, Dual targeting of mitochondrial proteins: mechanism, regulation and function, Biochim. Biophys. Acta Biomembr. 1808 (2011) 1012-1020.

[34] R. Rosenzweig, V. Bronner, D. Zhang, D. Fushman, M.H. Glickman, Rpn1 and Rpn2 coordinate ubiquitin processing factors at proteasome, J. Biol. Chem. 287 (2012) 14659-14671.

[35] J.M. Jones, J.C. Morrell, S.J. Gould, Identification and characterization of HAOX1, HAOX2, and HAOX3, three human peroxisomal 2-hydroxy acid oxidases, J. Biol. Chem. 275 (2000) 12590-12597.

[36] K.O. Haustein, G. Hüller, On the pharmacokinetics and metabolism of propiverine in man, Eur. J. Drug Metab. Pharmokinet. 13 (1988) 81-90.

[37] K. May, T. Giessmann, D. Wegner, R. Oertel, C. Modess, S. Oswald, M. Braeter, W. Siegmund, Oral absorption of propiverine solution and of the immediate and extended release dosage forms: influence of regioselective intestinal elimination, Eur. J. Clin. Pharmacol. 64 (2008) 1085-1092.

[38] W.M. Blankesteijn, H.L. Siero, J.F. Rodrigues de Miranda, Y.J. van Megen, F.G. Russel, Characterization of muscarinic receptors in rat kidney, Eur. J. Pharmacol. 244 (1993) 21-27.

[39] T. Haga, Molecular properties of muscarinic acetylcholine receptors, Proc. Jpn. Acad. B Phys. Biol. Sci. 89 (2013) 226-256.

[40] J. Hansen, S. Moriarty-Craige, D.P. Jones, Nuclear and cytoplasmic peroxiredoxin-1 differentially regulate NF-kappa B activities, Free Radic. Biol. Med. 43 (2007) 282-288.

[41] J.N. Rauch, J.E. Gestwicki, Binding of human nucleotide exchange factors to heat shock protein 70 (Hsp70) generates functionally Distinct complexes in vitro, J. Biol. Chem. 289 (2014) 1402-1414.

[42] G.B. Downes, N. Gautam, The G protein subunit gene families, Genomics 62 (1999) $544-552$.

[43] K. Kırlı, S. Karaca, H.J. Dehne, M. Samwer, K.T. Pan, C. Lenz, H. Urlaub, D. Görlich, A deep proteomics perspective on CRM1-mediated nuclear export and nucleocytoplasmic partitioning, eLife 4 (2015) e11466.

[44] T. Aoyama, K. Tsushima, M. Souri, T. Kamijo, Y. Suzuki, N. Shimozawa, T. Orii, T. Hashimoto, Molecular cloning and functional expression of a human peroxisomal acyl-coenzyme a oxidase, Biochem. Biophys. Res. Commun. 198 (1994) 1113-1118.

[45] S.M. Houten, S. Denis, C.A. Argmann, Y. Jia, S. Ferdinandusse, J.K. Reddy, R.J.A. Wanders, Peroxisomal L-bifunctional enzyme (EHHADH) is essential for the production of medium-chain dicarboxylic acids, J. Lipid Res. 53 (2012) 1296-1303.

[46] S. Miyazawa, S. Furuta, T. Osumi, T. Hashimoto, N. Ui, Properties of peroxisomal 3ketoacyl-CoA thiolase from rat liver, J. Biochem. 90 (1981) 511-519.

[47] P. Brites, H.R. Waterham, R.J.A. Wanders, Functions and biosynthesis of plasmalogens in health and disease, Biochim. Biophys. Acta Mol. Cell Biol. Lipids 1636 (2004) 219-231.

[48] K. Ghaedi, M. Honsho, N. Shimozawa, Y. Suzuki, N. Kondo, Y. Fujiki, PEX3 is the causal gene responsible for peroxisome membrane assembly-defective zellweger syndrome of complementation group G, Am. J. Hum. Genet. 67 (2000) 976-981.

[49] N. Hashiguchi, T. Kojidani, T. Imanaka, T. Haraguchi, Y. Hiraoka, E. Baumgart, S. Yokota, T. Tsukamoto, T. Osumi, Peroxisomes are formed from complex membrane structures in PEX6-deficient CHO cells upon genetic complementation, Mol. Biol. Cell 13 (2002) 711-722.

[50] S. Bar-Nun, M.H. Glickman, Proteasomal AAA-ATPases: structure and function, Biochim. Biophys. Acta Mol. Cell Res. 1823 (2012) 67-82.

[51] S.J. Steinberg, G.V. Raymond, N.E. Braverman, A.B. Moser, Peroxisome biogenesis Disorders - zellweger syndrome spectrum, in: Margaret P. Adam, Holly H. Ardinger, Roberta A. Pagon, Stephanie E. Wallace (Eds.), GeneReviews [Internet], University of Washington, Seattle, Seattle (WA), 2012.

[52] C.-Y. Fan, J. Pan, R. Chu, D. Lee, K.D. Kluckman, N. Usuda, I. Singh, A.V. Yeldandi, M.S. Rao, N. Maeda, J.K. Reddy, Hepatocellular and hepatic peroxisomal alterations in mice with a disrupted peroxisomal fatty acyl-coenzyme a oxidase gene, J. Biol. Chem. 271 (1996) 24698-24710.

[53] S.-H. Park, Y. Kukushkin, R. Gupta, T. Chen, A. Konagai, Mark S. Hipp, M. HayerHartl, F.U. Hartl, PolyQ proteins interfere with nuclear Degradation of cytosolic proteins by sequestering the Sis1p chaperone, Cell 154 (2013) 134-145.

[54] B. Fahrenkrog, U. Aebi, The nuclear pore complex: nucleocytoplasmic transport and beyond, Nat. Rev. Mol. Cell Biol. 4 (2003) 757-766.

[55] S.J. Gould, G.A. Keller, S. Subramani, Identification of peroxisomal targeting signals 
located at the carboxy terminus of four peroxisomal proteins, J. Cell Biol. 107 (1988) 897-905.

[56] M. Baum, F. Erdel, M. Wachsmuth, K. Rippe, Retrieving the intracellular topology from multi-scale protein mobility mapping in living cells, Nat. Commun. 5 (2014).

[57] S. Nagotu, V.C. Kalel, R. Erdmann, H.W. Platta, Molecular basis of peroxisomal biogenesis disorders caused by defects in peroxisomal matrix protein import, Biochim. Biophys. Acta (BBA) - Mol. Basis Dis. 1822 (2012) 1326-1336.

[58] J. Tyedmers, A. Mogk, B. Bukau, Cellular strategies for controlling protein aggregation, Nat. Rev. Mol. Cell Biol. 11 (2010) 777-788.

[59] M. Baes, P.P. Van Veldhoven, Hepatic dysfunction in peroxisomal disorders, Biochim. Biophys. Acta 1863 (2016) 956-970.

[60] A.H. Heussner, M.I. Baldock, M.I. Braeter, D.R. Dietrich, Characterization of propiverine effects on rat PPARs, Toxicol. Sci. 102 (01 suppl.) (2008) 93 no. 454.

[61] M. Yamasaki, N. Hashiguchi, C. Fujiwara, T. Imanaka, T. Tsukamoto, T. Osumi, formation of peroxisomes from peroxisomal ghosts in a peroxisome-deficient mammalian cell mutant upon complementation by Protein Microinjection, J. Biol.
Chem. 274 (1999) 35293-35296.

[62] L. Latonen, Nucleolar aggresomes as counterparts of cytoplasmic aggresomes in proteotoxic stress. Proteasome inhibitors induce nuclear ribonucleoprotein inclusions that accumulate several key factors of neurodegenerative diseases and cancer, Bioessays 33 (2011) 386-395.

[63] H. Inoue, M. Enomoto, K. Kobayashi, Two-year Oral Carcinogenicity Study in Mice (B6C3F1) with P4 (Propiverine Hydrochloride), Rep. No. 1556 (Apogepha ID P150), (1989) p. 258.

[64] S. Kohda, H. Nishikawa, M. Sumino, M. Sano, J. Kuroda, K. Miura, M. Tsuchitani, I. Narama, H. Yada, K. Yamashita, One-year chronic oral toxicity study of propiverine hydrochloride in dogs followed by one-month recovery, J. Toxicol. Sci. 14 (1989) 111-160.

[65] K. Yamashita, H. Yada, K. Irimura, A. Maruden, K. Morita, Thirteen-week oral toxicity study of propiverine hydrochloride in dogs, J. Toxicol. Sci. 14 (Suppl 2) (1989) 61-109. 